\section{A universal nucleoside for use at ambiguous sites in DNA primers}

\author{
R. Nlchols*†, P. C. Andrews*, P. Zhang $\$ \S$ \\ \& D. E. Bergstrom $₫ \S \|$
}

* Departments of Biological Chemistry and † Biology, University of Michigan, Ann Arbor, Michigan 48109, USA $\$$ Walther Cancer Institute, Indianapolis, Indiana 46208, USA $\S$ Department of Medicinal Chemistry and Pharmacognosy, Hansen Life Sciences Research Building, Purdue University, West Lafayette, Indiana 47907, USA

|| To whom correspondence should be addressed at Purdue University

A NON-DISCRIMINATORY base analogue, or universal base, would be an invaluable component of oligonucleotide probes and primers for solving the design problems that arise as a result of the degeneracy of the genetic code, or when only fragmentary peptide sequence data are available. We have designed an alternative to previous universal nucleoside candidates ${ }^{1-9}$, a new analogue, 1-(2'-deoxy- $\beta$ D-ribofuranosyl)-3-nitropyrrole (designated M; Fig. 1), which maximizes stacking while minimizing hydrogen-bonding interactions without sterically disrupting a DNA duplex. Oligonucleotides containing $M$ at several sites were used as primers for sequencing and the polymerase chain reaction. The sequencing primer $\mathrm{d}\left(5^{\prime}\right.$-CGT AAM CAM AAM ACM AT-3') is as effective as the exact match d(5'-CGT AAT CAG AAA ACA AT-3'). It is also possible to sequence using a primer containing $M$ at several contiguous positions, for example d(5'-CGT AAT MMM MMM MMM AT-3'). Melting curves show that duplexes formed on hybridization of the sequences d(5'-CCT TTT TMT TTT TGG-3') and d(5'-CCA AAA AXA AAA AGG-3'), where $X$ is $A, C, G$ or $T$, melted at a lower temperature than the corresponding duplexes containing only $d(A \cdot T)$ and $d(C \cdot G)$ base pairs, but showing little variation among different $\mathrm{X}$ bases $\left(T_{\mathrm{m}}\right.$ range $\left.3^{\circ} \mathrm{C}\right)$.

In our design and synthesis of nucleoside analogues, we aimed to maximize stacking interactions using aprotic polar substituents linked to heteroaromatic rings. Enhancing intra- and interstrand stacking interactions should lessen the role of hydrogen bonding in base-pairing specificity. Functional groups that would be expected to polarize a $\pi$-aromatic system and thereby enhance stacking, but which are not strong hydrogen-bond acceptors, include cyano and nitro groups. For maximum polarity and stacking we chose 3-substituted pyrrole deoxyribonucleosides as the best candidates for a first generation of weakly hydrogen-bonding universal nucleosides. Of the candidate molecules, we favoured 3-nitropyrrole 2'-deoxyribonucleoside (M; Fig. 1) because of its structural and electronic resemblance to $p$-nitroaniline, whose derivatives are among the smallest known intercalators of double-stranded $\mathrm{DNA}^{10}$. 3-Nitropyrrole $2^{\prime}$-de-

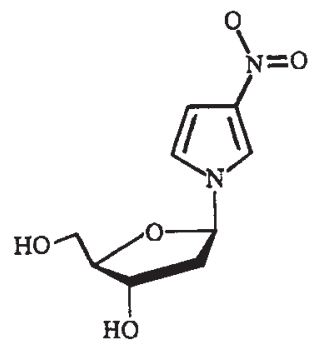

FIG. 1 Structure of 1 -(2'-deoxy- $\beta$-D-ribofuranosyl)-3-nitropyrrole. Details of the synthesis and crystal structure coordinates will be published elsewhere (D.E.B., P.Z., P.H.T., P.C.A. and R.N., manuscript in preparation). oxy-ribonucleoside was synthesized and its structure confirmed by X-ray crystallography. The dimethoxytrityl-protected phosphoramidite of nucleoside $M$ was synthesized for incorporation into olignucleotides used as primers for sequencing and polymerase chain reaction (PCR).

Sequencing studies show that a substantial number of nucleotides can be replaced by $M$ without loss of primer specificity (Fig. $2 a$ and $b$ ). Sequencing primer 4 with substitutions of $\mathbf{M}$ at the third position in each of four codons gave a sequencing ladder comparable to primer 1 , the exact match, whereas a 256 fold degenerate oligonucleotide mixture (primer 2) gave an unreadable sequencing ladder (Fig. 2a). Comparison of primers 3 and 4 is instructive as the former contains four deoxyinosines at the same sites occupied by $\mathbf{M}$ in primer 4 , and the sequencing ladder with primer 3 is only partially readable (Fig. $2 a$ ). In comparison, primers with two or more mismatches at the same

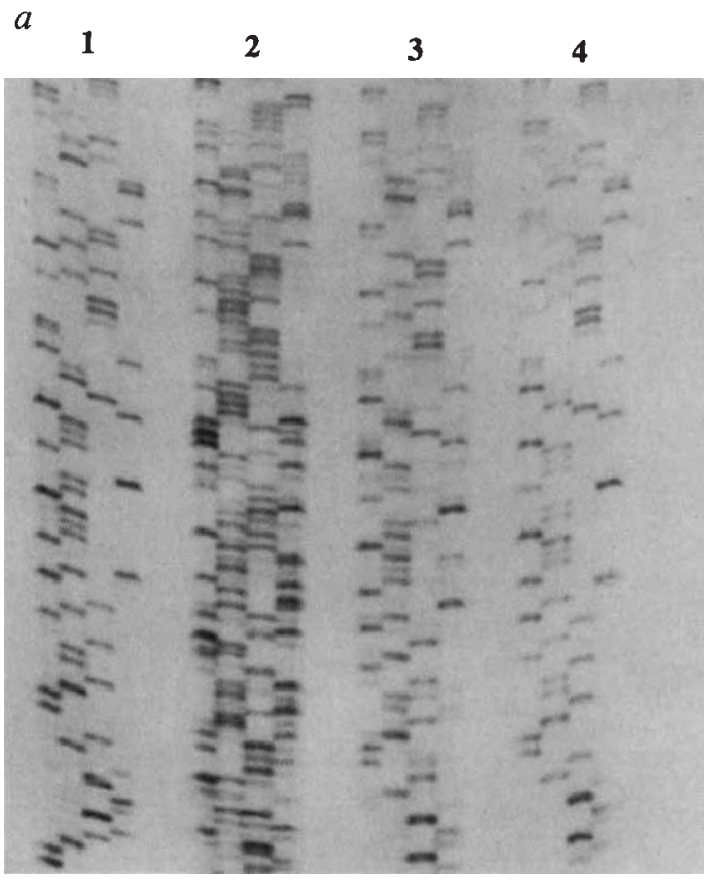

FIG. 2 a, Dideoxy sequencing using primers 1-4. Numbers correspond to the oligonucleotide sequencing primers $d\left(5^{\prime}\right.$-CGT AAT CAG AAA ACA AT- $\left.3^{\prime}\right)$ (primer 1); d( $5^{\prime}$-CGT AAN CAN AAN ACN AT-3'), where $N$ is A, C, $\mathrm{G}$ or T (primer 2); $\mathrm{d}\left(5^{\prime}\right.$-CGT AAI CAI AAI ACI AT-3') (primer 3); and d(5'CGT AAM CAM AAM ACM AT-3') (primer 4). The oligonucleotides were purified by HPLC on a $25 \mathrm{~cm} \times 4 \mathrm{~mm}$ polyhydroxyethyl aspartamide column (polyLC) or by denaturing-gel electrophoresis and size-exclusion chromatography. For sequencing, Sequenase version 2.0 (USB) was used according to the manufacturer's recommendations. Singlestranded DNA $(1 \mu \mathrm{g})$ from Drosophila melanogaster sulfakinin ${ }^{14}$ in Bluescript $\mathrm{SK}^{+}$(Stratagene) was sequenced using $0.1 \mu \mathrm{g}$ primer and ${ }^{35} \mathrm{~S}$-dATP (Amersham). Aliquots of sequencing reactions were electrophoresed on $6 \%$ acrylamide- $8 \mathrm{M}$ urea gels, and the gels dried and exposed to Kodak XAR X-ray film. b, Dideoxy sequencing using primers 1 and 57. Numbers correspond to the oligonucleotide sequencing primers $d\left(5^{\prime}-\right.$ CGT AAT CAG AAA MMM AT-3') (primer 5); d(5'-CGT AAT CAG MMM MMM AT-3') (primer 6); d(5'-CGT AAT MMM MMM MMM AT-3') (primer 7). Oligonucleotides were purified and sequenced as in $a$. The sequence ladder from primer 7 was consistently fainter than for primers 1,5 and 6 , resulting in overexposure of the first three lanes. C, PCR amplification using primers $1,4,8$ and 9 . Lane numbers correspond to the oligonucleotide primers. The sequence of primer 8 is d $\left(5^{\prime}\right.$-CGT AAT CAG AAA ACA AM- $\left.3^{\prime}\right)$, and primer 9 is d $\left(5^{\prime}-C G T\right.$ AAT CAG AAA ACM AT- $\left.3^{\prime}\right)$. The lower band in each lane arises from primer dimerization. The expected product is $\sim 315$ bases long. The identity of the product was verified by dideoxy sequence analysis (data not shown). First-strand synthesis was done using total RNA extracted from Drosophila melanogaster heads and avian myeloblastosis virus reverse transcriptase (Promega) and 
sites gave uninterpretable results. A unique property of $M$ is its ability to replace long strings of contiguous nucleosides and still yield functional sequencing primers. Sequences with three (primer 5), six (primer 6) and nine (primer 7) M substitutions (Fig. $2 b$ ) all gave readable sequencing ladders. PCR with primer 1 and three different $M$-containing primers (primers 4, 8,9) all resulted in amplification of the correct product (Fig. 2c).

The ability of 3-nitropyrrole-containing oligonucleotides to function as primers strongly suggests that a duplex structure must form with complementary strands. Optical thermal profiles obtained for the oligonucleotide pairs $\mathrm{d}\left(5^{\prime}-\mathrm{C}_{2} \mathrm{~T}_{5} \mathrm{XT}_{5} \mathrm{G}_{2}-3^{\prime}\right)$ and $\mathrm{d}\left(5^{\prime}-\mathrm{C}_{2} \mathrm{~A}_{5} \mathrm{YA}_{5} \mathrm{G}_{2}-3^{\prime}\right)$ (where $X$ and $Y$ can be $A, C, G, T$ or $M$ ) fit the normal sigmoidal pattern observed for the DNA doubleto-single strand transition. The melting temperatures $\left(T_{\mathrm{m}}\right)$ were determined at $260 \mathrm{~nm}$ in $1.0 \mathrm{M} \mathrm{NaCl}, 0.1 \mathrm{mM}$ EDTA, $10 \mathrm{mM}$ sodium phosphate, $\mathrm{pH} 7.0$, at an oligonucleotide concentration of $50 \mu \mathrm{M}$. The $T_{\mathrm{m}}$ values of the oligonucleotides containing X·M
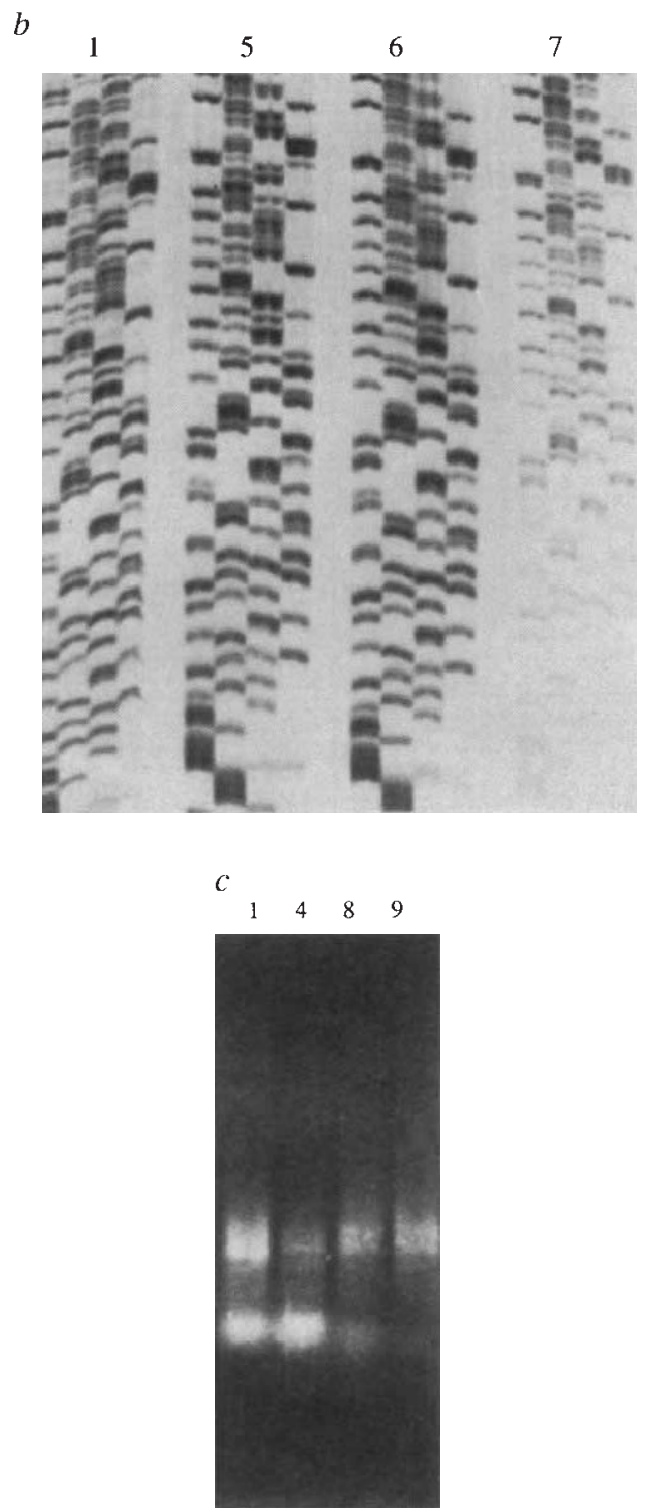

oligo(dT) at $42^{\circ} \mathrm{C}$ for $\sim 1 \mathrm{~h}$. The first-strand synthesis reaction was then divided into four aliquots and used for amplification with four different primers: 1, 4, 8 and 9. Each PCR was run for 30 cycles, with annealing at $48^{\circ} \mathrm{C}$, extension at $72^{\circ} \mathrm{C}$, and denaturing at $95^{\circ} \mathrm{C}$, using sequencespecific primer (primer 1, 4, 8 or 9), oligo(dT) and Taq polymerase (Perkin-EImer) according to the manufacturer's suggestions. Each reaction was analysed by gel electrophoresis and visualized by ethidium bromide staining. base pairs (where $\mathrm{X}$ was $\mathrm{A}, \mathrm{C}, \mathrm{G}$ or $\mathrm{T}$, and $\mathrm{Y}$ was $\mathrm{M}$ ) all fell within a $3{ }^{\circ} \mathrm{C}$ range. Deoxyinosine (dI), an extensively studied nucleoside with some charactertics of a universal nucleoside ${ }^{1 !}$, is more discriminating. Duplexes containing dI opposite any base vary in $T_{\mathrm{m}}$ by as much as $15^{\circ} \mathrm{C}$, corresponding to differences in base-pair stability of $2-3 \mathrm{kcal} \mathrm{mol}^{-1}$ (refs 12, 13).

This letter describes the first of a series of modified nucleosides which, in addition to their application as tools for use in molecular biology, should refine our understanding of the forces involved in DNA duplex formation and of the factors influencing specificity.

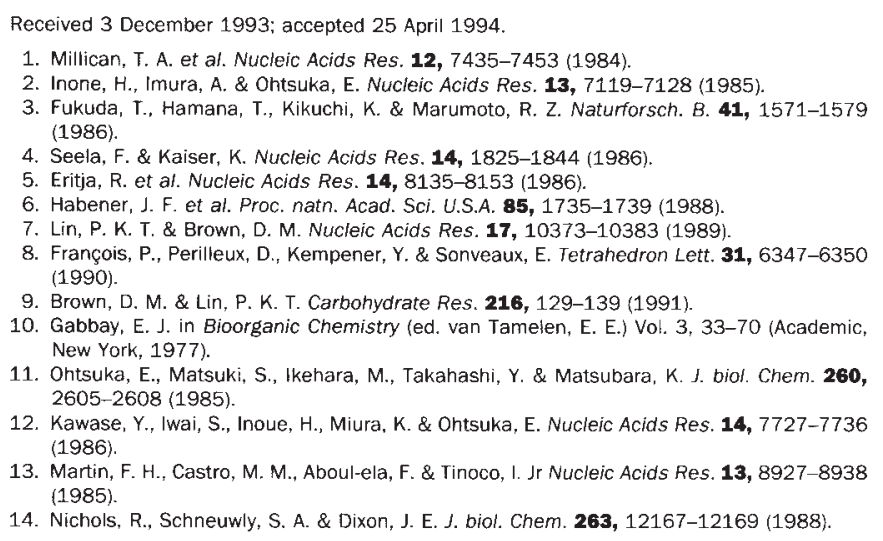

ACKNOWLEDGEMENTS. We thank M. F. Tibbetts for sequencing, H. Liu for PCR, and S. E. Olsen and T. A. Poley for $T_{m}$ studies. This work was supported by the NIH.

\section{The role of Rab3A in neurotransmitter release}

\section{Martin Geppert*†, Vadim Y. Bolshakovt, Steven A. Siegelbaum:, Kohji Takeiß, Pietro De Camilli $\$$, Robert E. Hammer $\dagger$ \& Thomas C. Südhof* ${ }^{*} \uparrow$}

* Departments of Molecular Genetics and

Biochemistry and $\dagger$ Howard Hughes Medical Institute,

The University of Texas Southwestern Medical Center, Dallas,

Texas 75235, USA

$\ddagger$ Department of Pharmacology. Center for Neurobiology and Behavior, Howard Hughes Medical Institute, Columbia University, New York, New York 10032, USA

$\S$ Department of Cell Biology and Howard Hughes Medical Institute, Yale University School of Medicine, New Haven,

Connecticut 06510, USA

THE small GTP-binding protein Rab3A is a Rab family member ${ }^{13}$ that is abundant in brain synaptic vesicles ${ }^{4,5}$. Here we show that mice in which the rab3A gene has been mutated by homologous recombination do not express Rab3A but are viable and fertile. Electrophysiological recordings in hippocampal CAI pyramidal cells indicate that most of their synaptic parameters are also normal, although synaptic depression after short trains of repetitive stimuli (15-30 stimuli at $14 \mathrm{~Hz})$ is significantly increased. Levels of the Rab3A-binding protein rabphilin are decreased by $70 \%$, but expression of more than 20 other synaptic proteins is unchanged. No compensatory changes were detected in other GTP-binding proteins or in proteins that interact with Rab3. Rab3A thus appears not to be essential for synaptic vesicle exocytosis but to play a role in the recruitment of synaptic vesicles for exocytosis during repetitive stimulation.

- To whom correspondence should be addressed. 